\title{
Evaluation of Lateral and Depth Resolutions of Light Field Cameras
}

\author{
Soweon Yoon ${ }^{1,3}$, Peter Bajcsy ${ }^{1}$, Maritoni Litorja ${ }^{2}$, and James J. Filliben ${ }^{1}$ \\ 1. Information Technology Laboratory, National Institute of Standards and Technology, Gaithersburg, \\ MD, USA \\ 2. Physical Measurement Laboratory, National Institute of Standards and Technology, Gaithersburg, \\ MD, USA \\ 3. Dakota Consulting Inc., Silver Spring, MD, USA
}

Light field cameras, also called plenoptic cameras, capture a field of light rays traveling in space, i.e., the intensity and direction of light rays. This is contrary to conventional still-picture cameras that acquire the aggregated intensity of incident light rays from all directions. A light field camera can be achieved by placing an array of microlenses between the imaging sensor and the main lens. Since a light field camera is essentially viewed as multiple cameras in a 2D array, it can function as a $3 \mathrm{D}$ camera based on multiple-view geometry. In crime scene investigations, 3D forensic evidence such as tire tread and shoe imprints in substances like mud or snow can often provide useful information to identify suspects and victims. This work focuses on evaluating lateral and depth resolutions of the light field cameras that are hand-held, easy to use, and affordable [1] to understand their suitability for forensic applications.

For the lateral resolution evaluation, the light field images of a resolution target plate with a 3 by 3 array of Siemens stars were collected to achieve a full factorial design with the following variables: (i) distance between the camera and the target plate $(330 \mathrm{~mm}$ to $1030 \mathrm{~mm}$ with a step size of $50 \mathrm{~mm})$, (ii) camera (two cameras from the same model), (iii) zoom level (level 1 for the field of view of $280 \mathrm{~mm}$, and level 2 for that of $195 \mathrm{~mm}$ ), and (iv) illumination (level 1 for ambient lighting, and level 2 with additional halogen lamps). Under each condition, five images were collected-local replicates-to observe the effect of random noise, for example, from the imaging sensor or the lamps. The entire data collection was repeated three times-global replicates - to observe any systematic errors coming from device setup and operation of the experiments. For the depth resolution evaluation, the images of a resolution target plate containing concentric circles with known spacing and thickness were collected. The resolution target plate was tilted at two known angles such that the depth of the points on the resolution target gradually changed from left to right. The rest of the experimental setup for the depth resolution evaluation was identical to the one for the lateral resolution evaluation. A total of 5400 light field images (1800 images for the lateral resolution evaluation and 3600 images for the depth resolution evaluation) were collected.

The lateral resolution was evaluated by a modulation transfer function (MTF) from the Siemens star, which measures the image contrast between black and white wedges in the Siemens star while the radius of a probe circle changes (see Fig. 1) [2]. The analysis results showed that (i) when a lower level of zoom was used, the lateral resolution tended to be stable regardless of distance between the camera and the target resolution plate, but when a higher level of zoom was used, it tended to decrease significantly with respect to distance, (ii) a higher zoom factor yielded a higher lateral resolution, (iii) two cameras of the same model did not show meaningful differences in lateral resolution, except for the difference in partial lens quality, (iv) the center region in the camera's field of view generally provided a better lateral resolution than the peripheral regions, and (v) the ambient lighting condition yielded a better lateral 
resolution than excessive illumination. While the results (i)-(iv) were largely expected, the result (v) was surprising. This result may imply that the increase in overall brightness of the image acquisition environment does not always improve the resolution power of the camera.

The depth resolution was evaluated by estimating the disparity between the points on the different concentric circles. The disparity is the movement of a scene point in the images when the camera viewpoint changes, and is represented as a slope in an epipolar-plane image (EPI) in the case of light field imaging [3]. It can be said that the depth resolution that is associated with the tilted angle of the resolution target plate is achieved if the disparity estimation along the horizontal line of the resolution plate shows a monotonic trend (see Fig. 2). The preliminary results showed that depth difference less than $1 \mathrm{~mm}$ was resolvable.

The datasets for the evaluation of lateral and depth resolutions of the light field cameras and the analysis results are available online with an interactive user interface [4,5].

\section{References:}

[1] Lytro, Inc., https://www.lytro.com/.

[2] ISO 12233, "Photography - Electronic still picture imaging - Resolution and spatial frequency responses", (2017).

[3] R Bolles et al, "Epipolar-plane image analysis: An approach to determining structure from motion", International Journal of Computer Vision 1 (1987), p. 7-55.

[4] https://isg.nist.gov/deepzoomweb/resources/lytroEvaluations/index.html

[5] Commercial products are identified in this document in order to specify the experimental procedure adequately. Such identification is not intended to imply recommendation or endorsement by the National Institute of Standards and Technology, nor is it intended to imply that the products identified are necessarily the best available for the purpose.

(a)

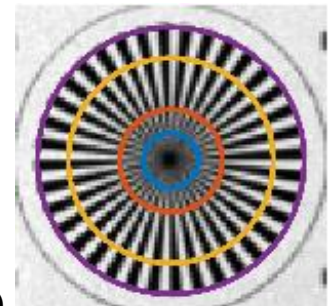

(b)

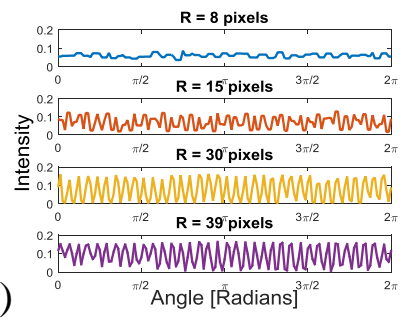

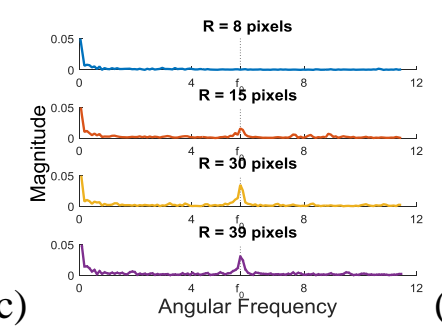

(c)

(d)

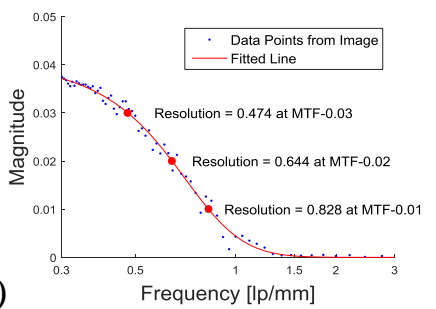

Figure 1. Measurement of lateral resolution. (a) Siemens star with four probe circles, (b) image intensity profile along each of the four circles, (c) Fourier transform of image intensity profiles, and (d) MTF.

(a)

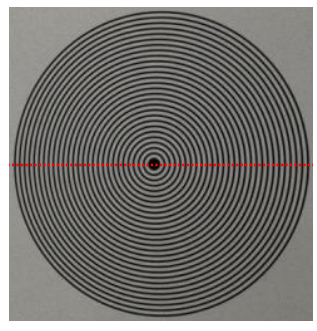

(b)

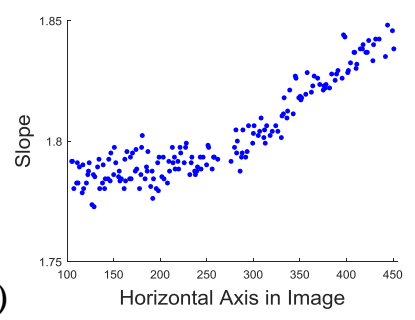

(c)

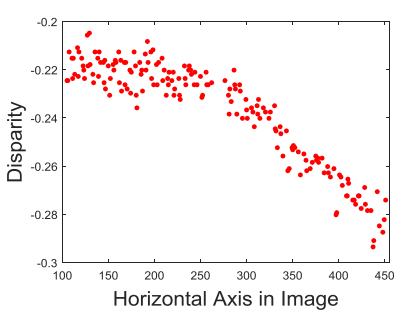

Figure 2. Measurement of depth resolution. (a) Concentric circles on a tilted plate, (b) slope estimation in EPI along the red line in (a), and (c) disparity estimation along the red line in (a). 